\title{
An unresectable gastric cancer with bulky lymph node metastases treated with SOX chemotherapy plus apatinib followed by D3 radical gastrectomy: a case report
}

\author{
Nannan Zhang ${ }^{1 \#}$, Jingyu Deng ${ }^{1 \#}$, Yan Sun ${ }^{2,3}$, Jianyu Xiao $^{3,4}$, Hongli Li $^{3,5}$, Han Liang ${ }^{1}$ \\ ${ }^{1}$ Department of Gastroenterology, Tianjin Medical University Cancer Hospital, City Key Laboratory of Tianjin Cancer Center and National \\ Clinical Research Center for Cancer, Tianjin, China; ${ }^{2}$ Department of Pathology, Tianjin Medical University Cancer Institute and Hospital, National \\ Clinical Research Center for Cancer, Key Laboratory of Cancer Prevention and Therapy, Tianjin, China; 'Tianjin's Clinical Research Center for \\ Cancer, Tianjin Medical University, Ministry of Education, Tianjin, China; ${ }^{4}$ Department of Radiology, Tianjin Medical University Cancer Institute \\ and Hospital, National Clinical Research Center for Cancer, Key Laboratory of Cancer Prevention and Therapy, Tianjin, China; ${ }^{5}$ Department of \\ Gastrointestinal Oncology, Tianjin Medical University Cancer Institute and Hospital, National Clinical Research Center for Cancer, Key Laboratory \\ of Cancer Prevention and Therapy, Tianjin, China \\ \#These authors contributed equally to this work. \\ Correspondence to: Jingyu Deng, MD. Department of Gastric Cancer, Tianjin Medical University Cancer Institute \& Hospital, National Clinical \\ Research Center of Cancer, Key Laboratory of Cancer Prevention and Therapy, Tianjin's Clinical Research Cancer for Cancer, Tianjin 300060, \\ China. Email: dengery@126.com; Han Liang, MD. Department of Gastric Cancer, Tianjin Medical University Cancer Institute \& Hospital, \\ National Clinical Research Center of Cancer, Key Laboratory of Cancer Prevention and Therapy, Tianjin's Clinical Research Cancer for Cancer, \\ Tianjin 300060, China. Email: tjlianghan@126.com.
}

\begin{abstract}
Conversion therapy for gastric cancer (GC) has been the subject of much recent attention. GC patients with bulky lymph node metastases were usually considered oncologically unresectable and surgery could be challenging and tumor shrinkage may serve to facilitate resection. Previous studies reported satisfactory survival data were obtained in the series of neoadjuvant studies with bulky $\mathrm{N}$ disease. However, the evidence of combining neoadjuvant chemotherapy with targeted therapy for patients with bulky $\mathrm{N}$ disease is inadequate. We report a 52 -year-old man who was diagnosed with unresectable GC with bulky lymph node metastases after endoscopic biopsy and abdominal enhanced computed tomography (CT) examination. Histopathology confirmed poorly differentiated adenocarcinoma at the junction of the antrum and the body of the stomach. Abdominal enhanced CT showed marked thickening of more than two-thirds of the stomach wall and multiple enlarged and coalesced perigastric and extragastric lymph nodes. The clinical staging was cT4aN3M0. The patient was administered two cycles of S-1 and oxaliplatin (SOX regimen) plus apatinib. Repeat abdominal enhanced CT demonstrated decrease in stomach wall thickness and in the sizes of all perigastric and extragastric lymph nodes $(<1.0 \mathrm{~cm})$. D2 gastrectomy with para-aortic lymph node dissection was performed after 5 weeks. Pathological examination of resected specimen revealed a ypT4bN0M0 poorly differentiated adenocarcinoma. All 140 lymph nodes that were examined were negative. SOX chemotherapy regime was advised after surgery, but had to be discontinued after two cycles because of severe side effects. The patient has been followed up regularly for more than 2 years with enhanced abdominal CT and the examination of tumor markers. No recurrence or metastasis has been identified till the time of submission of this article. Our treatment experience might provide a reference for the treatment of GC patients with bulky lymph node metastases.
\end{abstract}

Keywords: Stomach; neoplasms; chemotherapy; apatinib; gastrectomy; case report

Submitted Mar 08, 2020. Accepted for publication Dec 04, 2020.

doi: $10.21037 /$ tcr-20-1400

View this article at: http://dx.doi.org/10.21037/tcr-20-1400 


\section{Introduction}

Gastric cancer (GC) remains the second most common cause of cancer-related death worldwide despite the steady decrease in its incidence over the past decades (1). There is consensus that curative resection is the only way to achieve cure in GC and that perioperative chemotherapy might help prolong overall survival (OS) in patients with advanced stage disease (2). Conversion therapy is adopted to achieve radical cure for patients with originally unresectable but potentially resectable GC, who obtain partial or complete remission after systemic treatment, to acquire relatively longer postoperative survival and recurrence-free survival. GC patients with bulky N2 disease (i.e., with at least three second-tier lymph nodes measuring $\geq 1.5 \mathrm{~cm}$ or with a mass measuring $\geq 3 \mathrm{~cm}$ composed of two or more secondtier lymph nodes) have relatively poorer prognosis and shorter survival (3). Moreover, GC with bulky lymph node metastases were usually considered oncologically unresectable.

The most efficient preoperative chemotherapy regimen currently available for advanced stage GC appears to be the combination of fluorouracil and platinum drugs (4-7). Apatinib, a novel, small molecule, selective vascular endothelial growth factor receptor-2 (VEGFR-2) tyrosine kinase inhibitor, may significantly improve OS and progression-free survival (PFS) in patients with advanced stage GC refractory to two or more prior chemotherapies (8). With regard to surgery, the latest edition of the Japanese Guidelines for Gastric Cancer recommends D2 lymphadenectomy as the standard procedure for GC patients, although other sources suggest that D2 lymphadenectomy plus para-aortic lymph node dissection could improve prognosis in some specific subgroups (9).

GC patients with bulky N2 disease (i.e., with at least three second-tier lymph nodes measuring $\geq 1.5 \mathrm{~cm}$ or with a mass measuring $\geq 3 \mathrm{~cm}$ composed of two or more second-tier lymph nodes) have relatively poorer prognosis and shorter survival (3). However, a recent study that analyze two phase II trials (JCOG0001 and JCOG0405) showed that the prognosis of GC patients with bulky N2 lymph node metastasis is still poor and further treatment improvement is needed (10).

We report a patient with unresectable GC and bulky lymph node metastases who was treated with two cycles of S-1 and oxaliplatin (SOX) chemotherapy plus apatinib, followed by curative gastrectomy and D2 gastrectomy with para-aortic lymph node dissection. There was no tumor recurrence or distant metastasis even 2 years after surgery. This treatment of combining neoadjuvant chemotherapy with targeted therapy for unresectable GC patients with bulky lymph node metastases might provide a treatment reference. We present the following article in accordance with the CARE reporting checklist (available at http:// dx.doi.org/10.21037/tcr-20-1400).

\section{Case presentation}

A 52-year-old man suffering from intermittent melena and epigastric discomfort for 3 months was diagnosed with unresectable GC and bulky lymph node metastases after endoscopic biopsy and abdominal enhanced computed tomography (CT) examination. He did not smoke, drink alcohol, or use intravenous drugs. Endoscopic ultrasonography revealed an ulcer-type tumor, with maximum diameter of $4.0 \mathrm{~cm}$, involving more than twothirds of the stomach wall between the antrum and the body of the stomach (Figure 1A,B). Histopathological examination of biopsy specimen by two pathologists confirmed poorly differentiated adenocarcinoma (Figure 1C). Abdominal enhanced CT showed diffuse thickening of the stomach wall and multiple enlarged and coalesced perigastric and extragastric lymph nodes (Figure 2). Serum tumor markers were within normal limits (CA-242: 1.50 IU/mL, CA72-4: $1.19 \mathrm{U} / \mathrm{mL}$, CA19-9: 7.51 U/mL, CEA: $4.26 \mu \mathrm{g} / \mathrm{L}$ ). The patient was diagnosed as unresectable GC case with cT4bN3M0 stage.

After multidisciplinary team (MDT) consultation, it was decided to administer three cycles of systemic SOX chemotherapy plus apatinib, and then repeat abdominal enhanced CT scan to reassess the feasibility of curative gastrectomy. Apatinib was given orally $(500 \mathrm{mg} /$ day) for continuous two cycles, followed by a one-cycle rest. S-1 was given orally twice daily for 2 weeks, followed by a 1-week break. The dose of S- 1 was determined based on the body surface area (BSA) as follows: BSA $<1.25 \mathrm{~m}^{2}, 80 \mathrm{mg} /$ day; BSA 1.25 to $<1.50 \mathrm{~m}^{2}, 100 \mathrm{mg} /$ day and BSA $1.50 \mathrm{~m}^{2}$, $120 \mathrm{mg} /$ day. And this patient was administrated with the dose of $100 \mathrm{mg} /$ day for his BSA between 1.25 and $1.50 \mathrm{~m}^{2}$. Oxaliplatin was administered at $130 \mathrm{mg} / \mathrm{m}^{2}$ intravenously on day 1. However, the patient was only treated for two cycles due to the severe side effects. Repeat enhanced CT after drug therapy showed partial remission of the stomach wall thickness, and decrease in the maximum diameter of all perigastric and extragastric lymph nodes to $<1.0 \mathrm{~cm}$ 

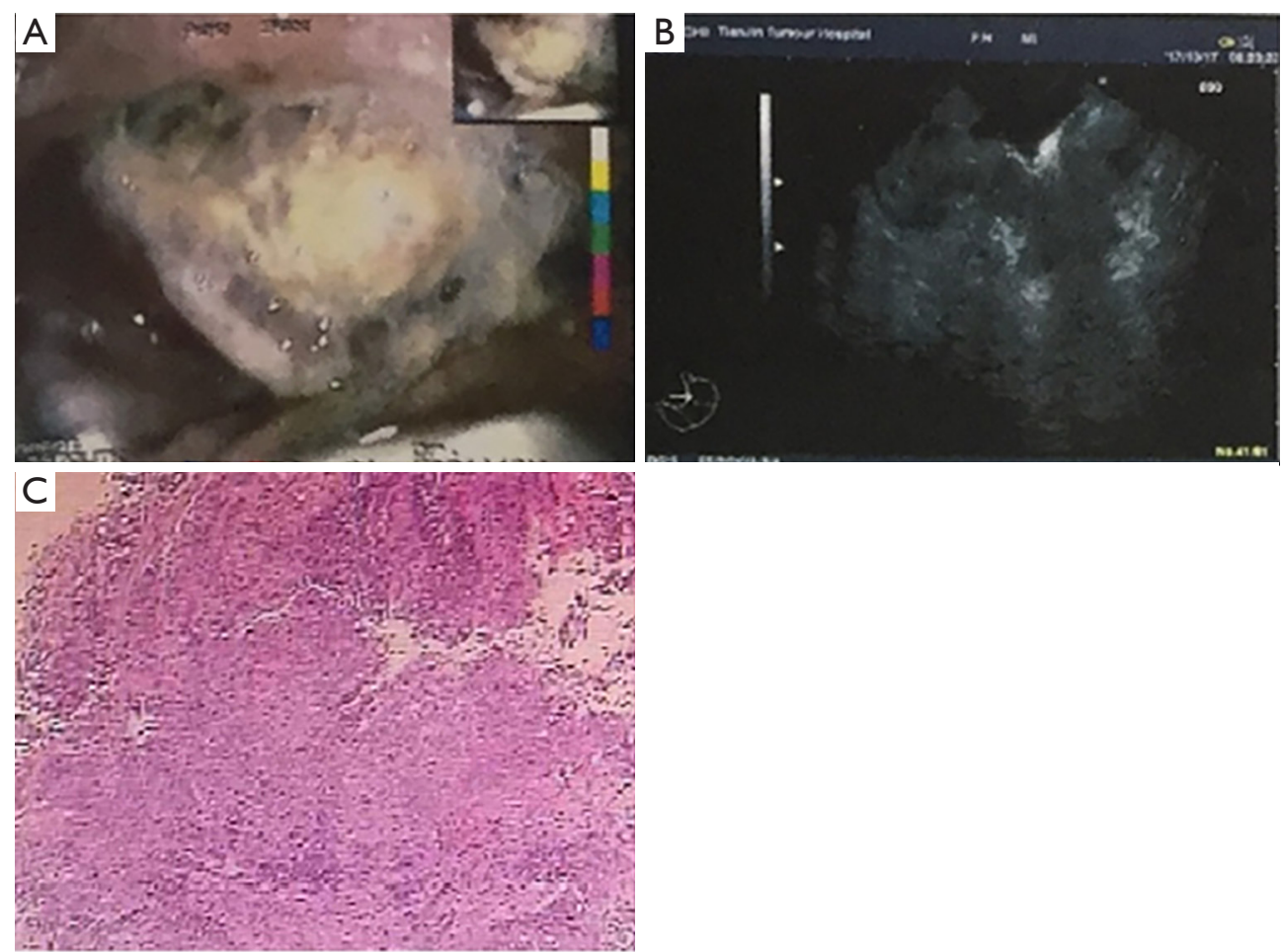

Figure 1 Preoperative examination results. (A) The endoscopic examination of primary tumor in stomach before surgery; (B) the ultrasonography examination of primary tumor in stomach before surgery; $(\mathrm{C})$ the pathological result of primary tumor in stomach before surgery $(\mathrm{HE}, \mathrm{SP} \times 100)$.
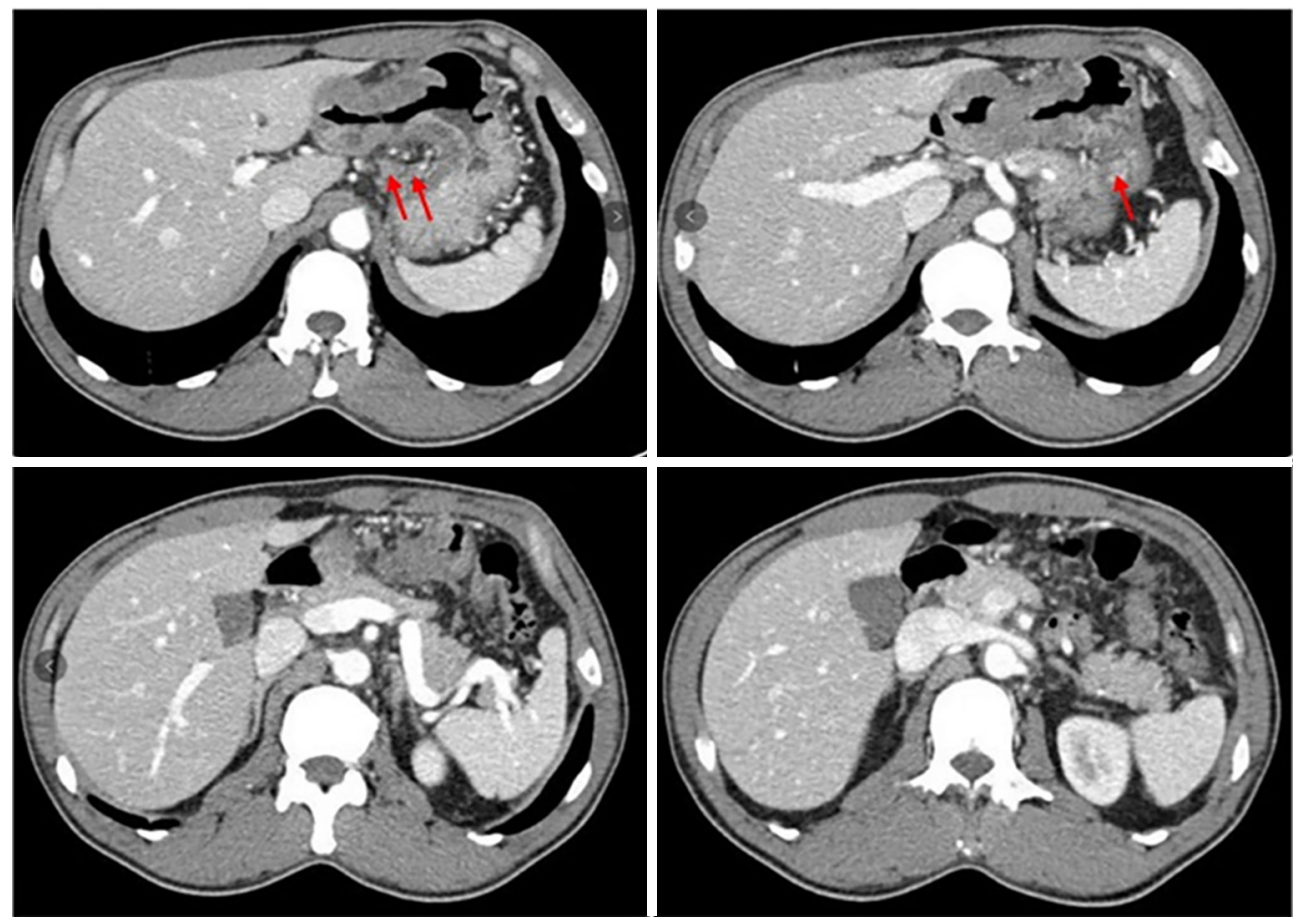

Figure 2 The abdominal enhanced CT scanning showed the significantly diffused thickness of stomach wall with multiple enlarged and coalesced perigastric and extragastric lymph nodes (Bulky lymph nodes labeled by the red arrow). CT, computed tomography. 

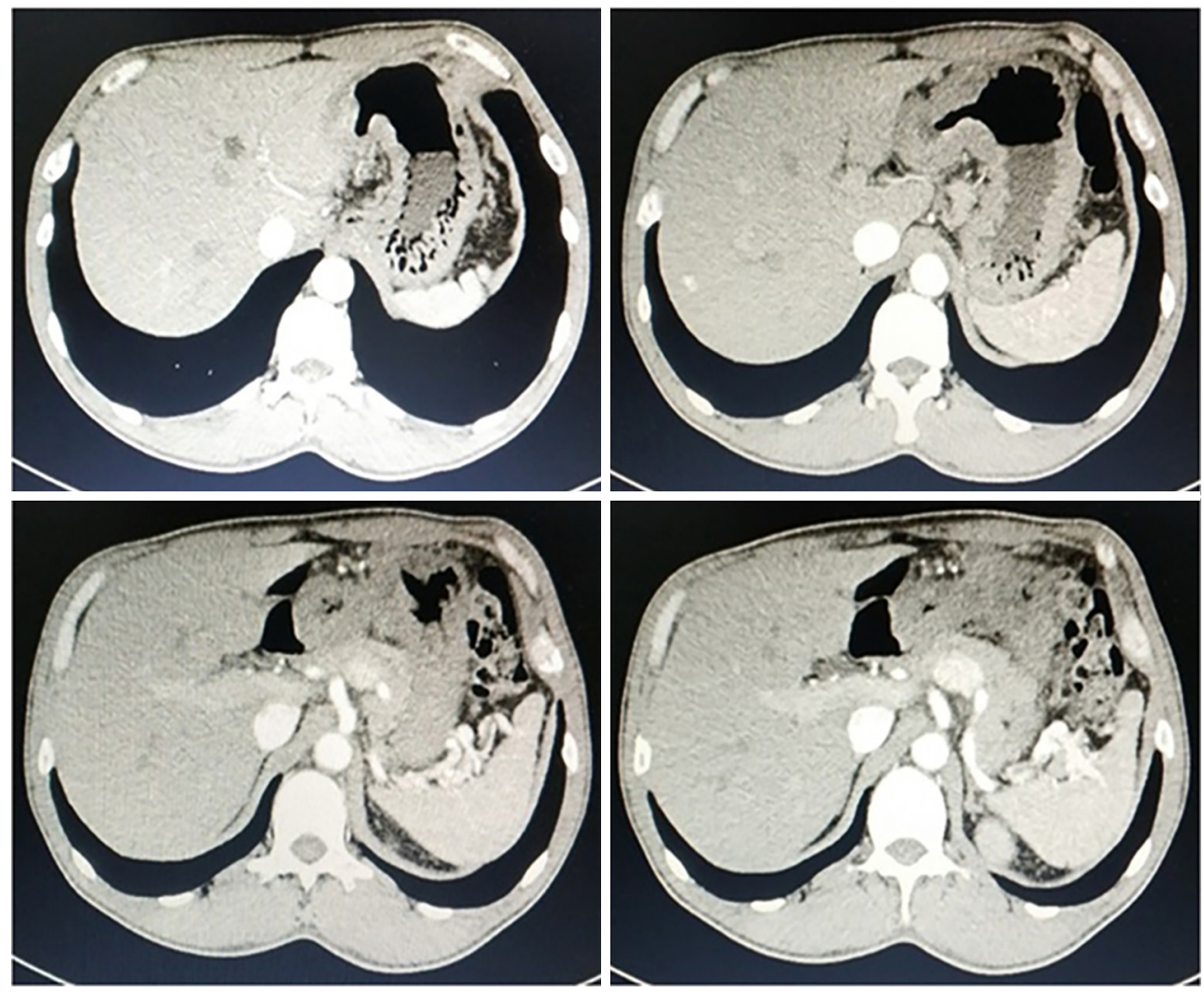

Figure 3 The abdominal enhanced CT scanning demonstrated that partial remission of the stomach wall thickness and the perigastric and extragastric lymph nodes after two cycles of SOX regimens chemotherapy plus apatinib. CT, computed tomography; SOX, S-1 and oxaliplatin.

(Figure 3). Tumor markers were not markedly increased (CA-242: 1.72 IU/mL, CA72-4: 1.34 U/mL, CA19-9: 9.67 U/mL, CEA: $6.15 \mu \mathrm{g} / \mathrm{L})$.

Four weeks later, after peritoneal dissemination was ruled out by laparoscopy (Figure $4 A$ ) and peritoneal lavage cytology (Figure 4B), radical distal subtotal gastrectomy plus D2 lymphadenectomy with para-aortic lymph node dissection was performed (Figure 5). The ulcer-type tumor was located in the lesser curvature of stomach body. Serosal and transverse mesocolic invasion was present. Histopathological examination confirmed poorly differentiated adenocarcinoma. However, all 140 examined lymph nodes were negative. The chemotherapy response of the tumor was TRG 3 grade, and the TNM classification was ypT4bN0M0. Expression of cancer molecular biomarkers on immunohistochemical staining was as follows: CK8/18 (+), CK7 (+), Ki-67 (80\%), and HER-2 (-) (Figure 6).

The patient was discharged 10 days after surgery.
Two cycles of postoperative SOX chemotherapy were administered, but then had to be stopped because of serious side effects. The patient has been followed up regularly with CT scan and endoscopy. No local recurrence or distant metastasis has been detected even 2 years after surgery (Figure 7), and serum tumor markers remain within normal limits (CA72-4 1.15 U/mL, CA19-9 2.38 U/mL, CA125 $3.88 \mathrm{U} / \mathrm{mL})$.

All procedures performed in studies involving human participants were in accordance with the ethical standards of the institutional and/or national research committee(s) and with the Helsinki Declaration (as revised in 2013). Written informed consent was obtained from the patient. The timeline of case report is clearly shown in Figure 8.

\section{Discussion}

Conversion therapy, a new treatment strategy for GC, is attracting wide attention. Some retrospective analyses have 

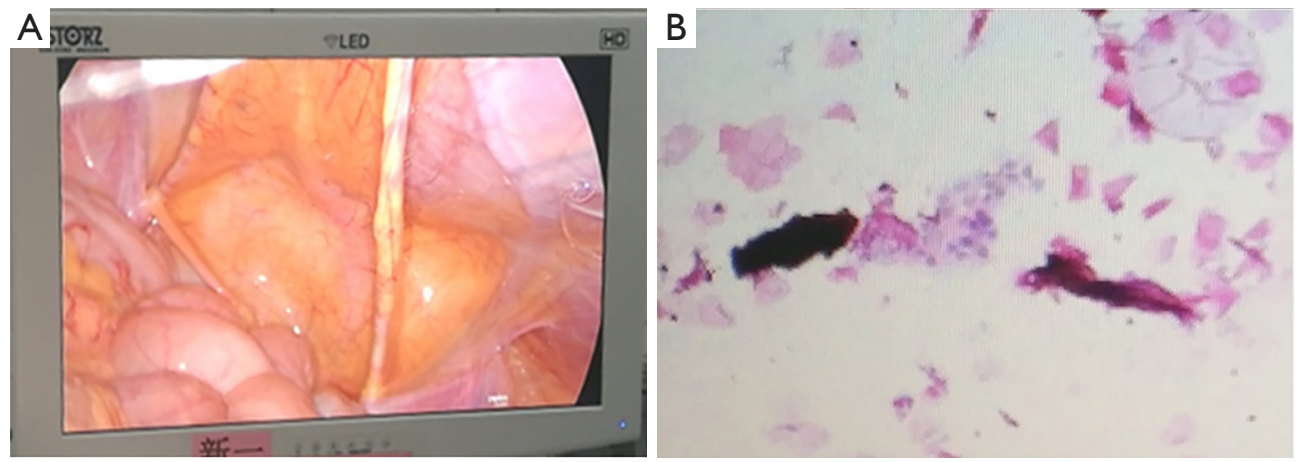

Figure 4 Intraoperative diagnosis. (A) The laparoscopic detection of the abdominopelvic cavity of patient; (B) the result of the peritoneal lavage cytology of patient (SP $\times 100$, HE staining).
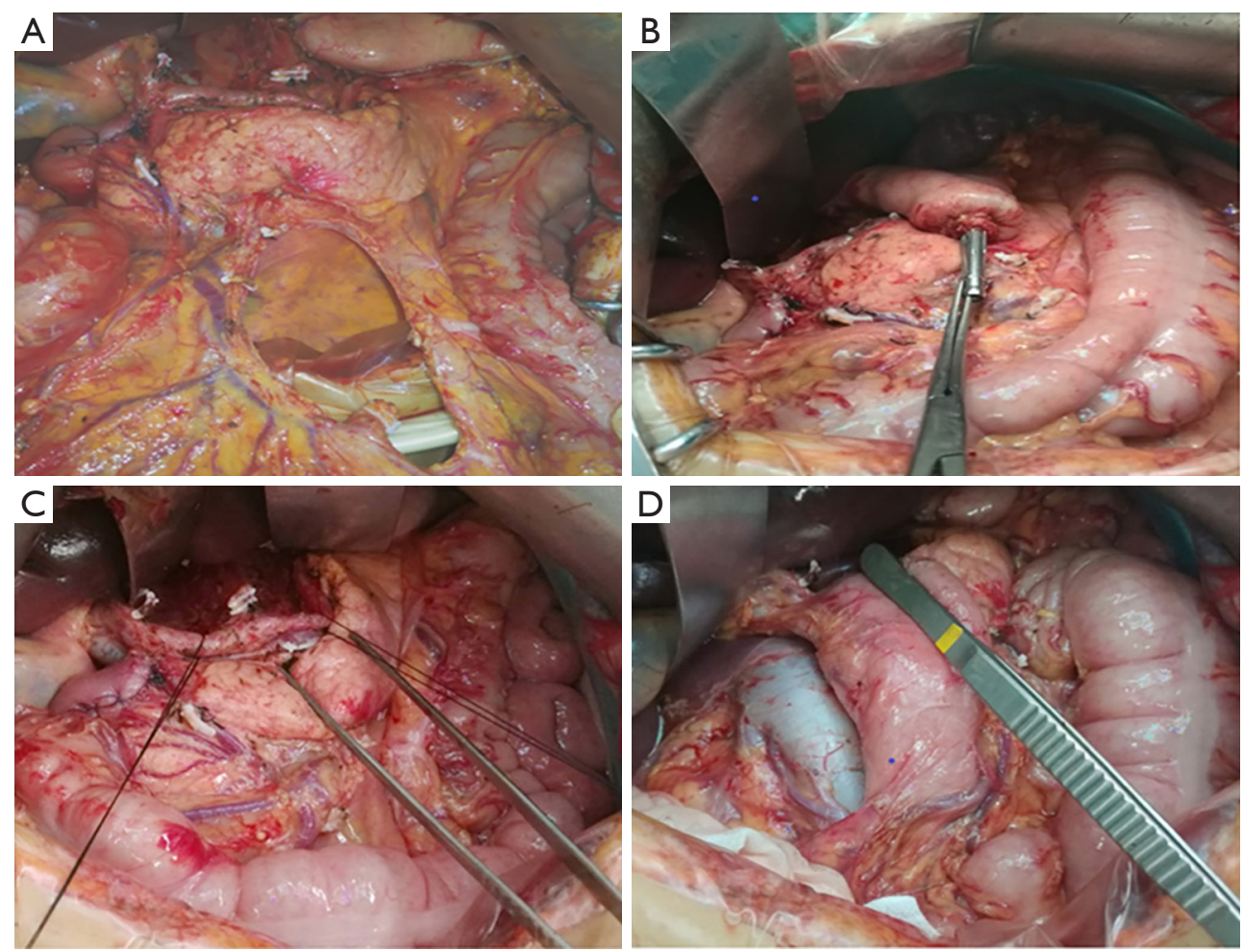

Figure 5 Intraoperative operation image. (A) Dissection of the segment of transverse mesocolon invaded by the primary tumor; (B) distal subtotal gastrectomy was performed; (C) D2 lymphadenectomy was accomplished in the procedure of curative gastrectomy for this patient; (D) the para-aortic lymph node dissection was performed for this patient.

revealed that S-1 based treatment regimens can provide good prognosis for patients in whom conversion therapy is successful $(11,12)$. Sato et al. (12) reported that docetaxel, cisplatin, and S-1 (DCS) chemotherapy followed by surgery could improve long-term survival, especially in patients with lymph node metastases or T4 stage disease (invasion of an adjacent organ). In Japan, after a long struggle and with support from some compelling evidence from the JCOG phase II studies on bulky N disease, Kodera (3) demonstrated that S-1 and cisplatin can be the standard first-line neoadjuvant chemotherapy for advanced GC with bulky lymph node metastases. Preoperative chemotherapy 

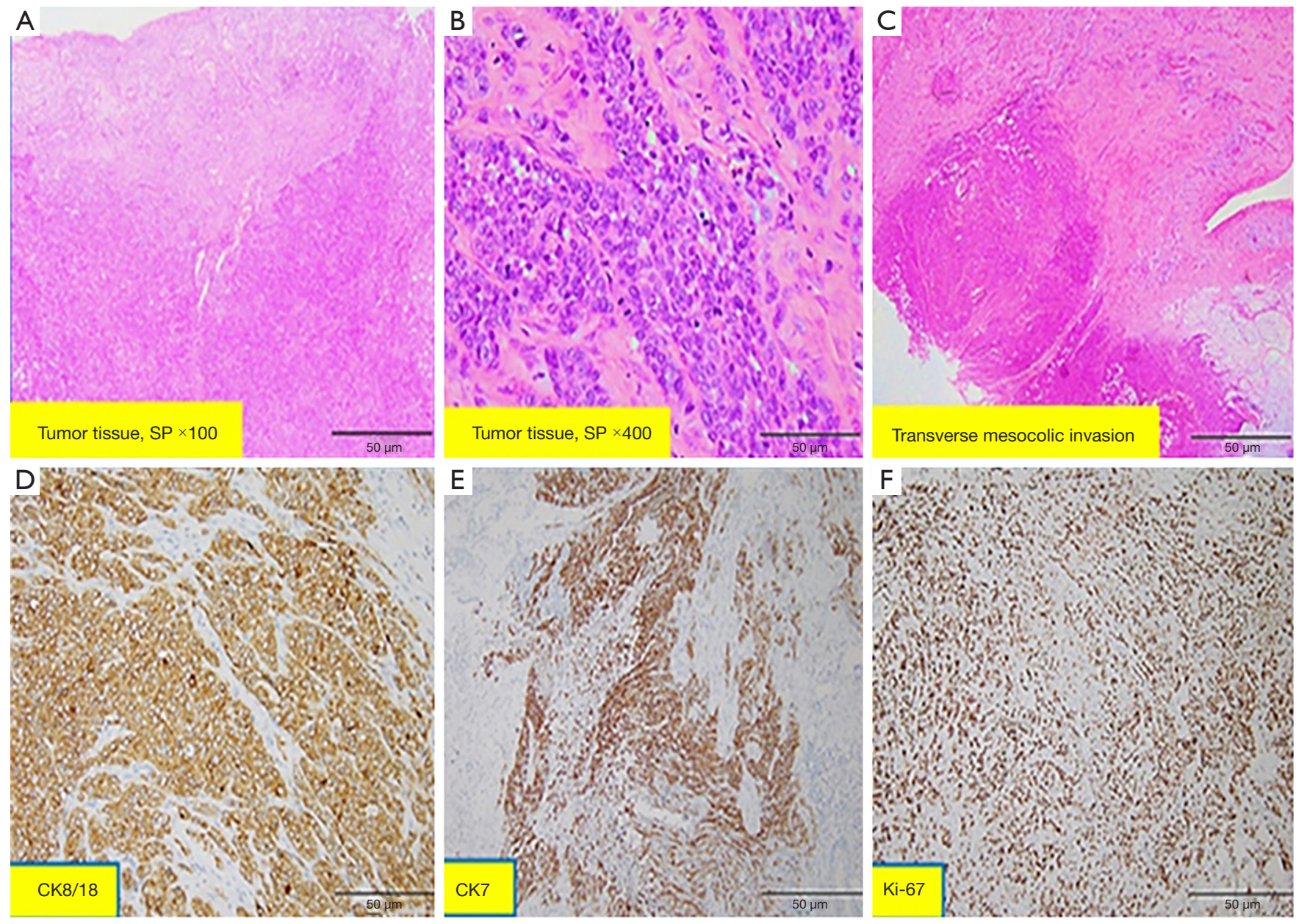

Figure 6 The postoperative histopathological result of the patient. (A,B,C) HE staining; (D,E,F) IHC staining.

has several benefits: it increases the probability of curative resection, it eliminates micrometastasis, it provides rapid improvement in tumor-related symptoms, and it determines the chemosensitivity of the tumor $(13,14)$. In the latest edition of the Japanese Gastric Cancer Treatment guidelines, both SOX are recommended for prolonging survival in advanced stage GC patients (4-6). Oxaliplatin was proved to be less nephrotoxic than cisplatin. Moreover, Combination chemotherapy with SOX for advanced GC has shown promising efficacy with acceptable toxicity $(15,16)$ and was recommended as neoadjuvant chemotherapy for patients with advanced GC in previous studies (17). Nevertheless, a recent integrated analysis of two phase II trials (JCOG0001 and JCOG0405) of preoperative chemotherapy for unresectable GC showed that poor prognosis in patients having both bulky $\mathrm{N}+$ and PAN+ may necessitate further treatment improvement (10).
Apatinib, a new VEGFR-2 tyrosine kinase inhibitor that targets the intracellular ATP binding site of the receptor, inhibits VEGF-mediated proliferation, invasion, migration, and angiogenesis of the cancer (18). The combination of apatinib plus cisplatin has been demonstrated to inhibit tumor growth and improve survival in many human malignancies (8). Furthermore, a recent study showed that SOX plus apatinib followed by surgery in patients with locally advanced gastric adenocarcinoma showed favorable activity and manageable safety (19). In our patient, abdominal enhanced CT scan performed after two cycles of SOX chemotherapy plus apatinib demonstrated marked decrease in the thickness of the stomach wall and in the sizes of perigastric and extragastric lymph nodes.

D2 gastrectomy is currently the standard surgical procedure for advanced stage GC. In Japan, regional lymph node dissection is still regarded as the cornerstone of radical 

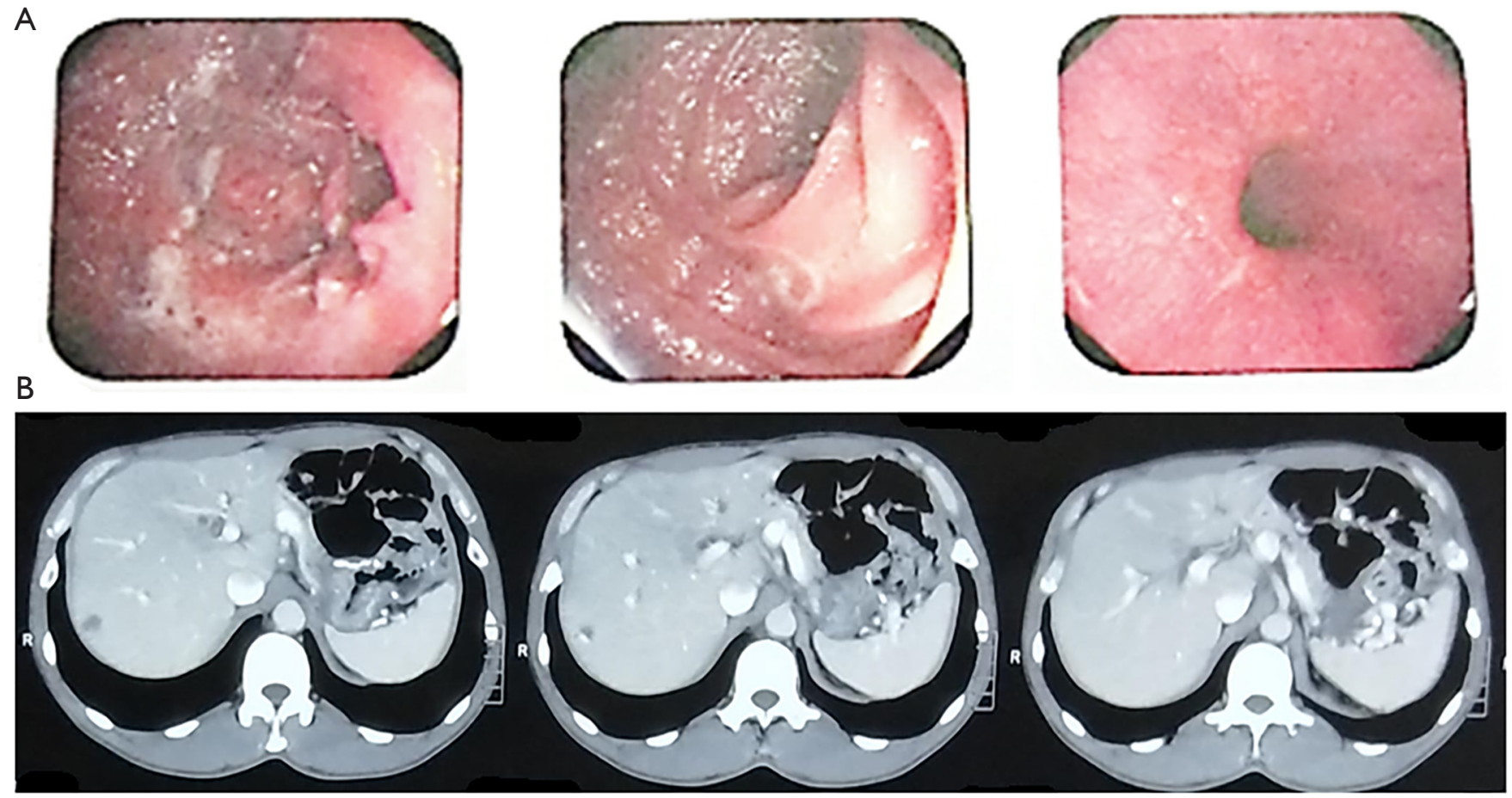

Figure 7 Postoperative follow-up examination results. (A) The endoscopic examination showed no evidence of recurrence in remnant stomach or in anastomosis; (B) the abdominal enhanced CT scanning showed no evidence of recurrence and distant metastasis. CT, computed tomography.

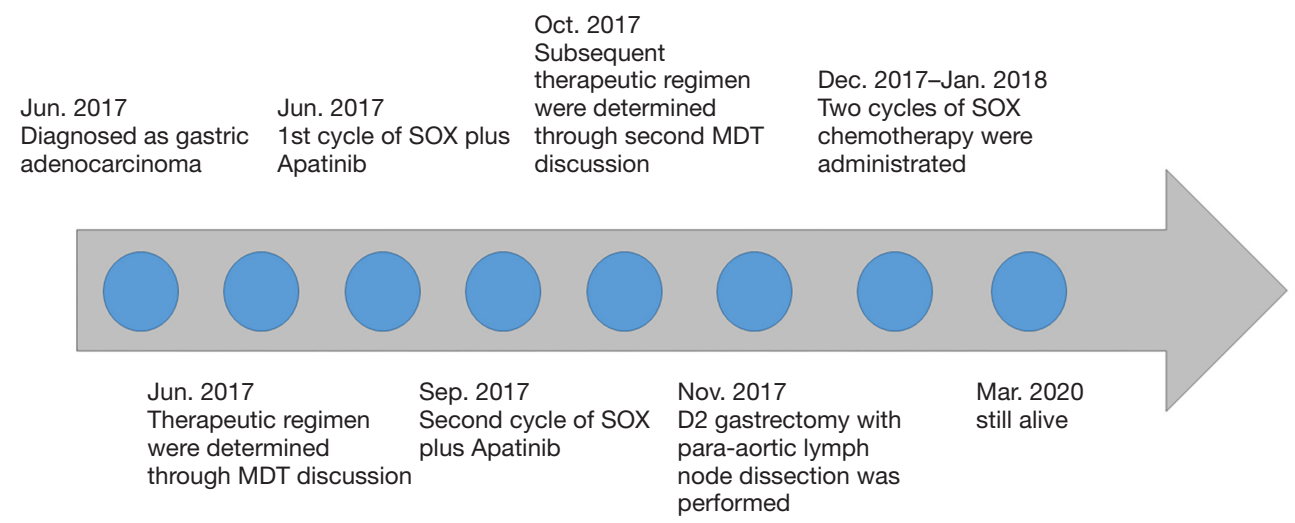

Figure 8 Case report timeline. SOX, S-1 and oxaliplatin; MDT, multidisciplinary team.

gastrectomy, as some resectable tumors may be considered as "locoregional lesions" in principle $(20,21)$. Many advanced stage GC patients have high risk of cancer cells spreading among the para-aortic lymph nodes in theory, which might not be eradicated by D2 lymphadenectomy. Several investigators have reported that 5-year survival for N2 stage patients is slightly better with D2 lymphadenectomy plus para-aortic lymph node dissection than with D2 lymphadenectomy alone (21). Others have shown that super-extended lymph node dissection (D3) could induce stage migration of lymph node metastasis to indirectly affect the OS of GC patients (22). Recently, three clinical trials (JOCG0001, JOCG0405, and JOCG1002) demonstrated that D2 lymphadenectomy plus para-aortic lymph node dissection could improve 5-year survival after curative resection for GC; the investigators concluded that 
this should be the recommended procedure for GC patients with bulky lymph node metastasis (3).

In our patient, the histopathological response to the conversion treatment was TRG 3 grade, indicating at least partial benefit from neoadjuvant treatment (23). Thus, the SOX plus apatinib regimen may have been effective in suppressing metastasis. A multicenter phase II study of apatinib treatment for metastatic GC indicated that the drug is effective and safe in patients who failed to respond to two or more prior chemotherapy regimens, and that the toxicities were clinically manageable (24). A systematic review and meta-analysis showed that apatinib plus chemotherapy is more effective than chemotherapy alone for patients with advanced GC (25). Dong et al. (26) demonstrated that SOX is more effective than fluorouracil, leucovorin, oxaliplatin (FOLFOX6) and capecitabine, oxaliplatin (XELOX) for controlling lymph node metastasis in locally advanced GC before surgery; the extragastric lymph node metastatic rates with the three chemotherapy regimens were $26.9 \%$ vs. $52.2 \%$ vs. $42.1 \%$, respectively.

To conclude, our experience with the patient reported here suggests that neoadjuvant therapy with SOX and apatinib can inhibit lymph node metastasis and thereby improve prognosis after surgery in patients with advanced stage GC. Our treatment experience might provide a reference for the treatment of GC patients with bulky lymph node metastases. However, the limitation of this study is that this case report only provided the treatment experience of one patient and whether this treatment is effective for all advanced GC is unknown. Our ongoing randomized controlled trial of this neoadjuvant approach in patients with unresectable GC will provide more evidence.

\section{Acknowledgments}

Funding: This study was supported in part by grants from the Programs of National Natural Science Foundation of China (No. 81572372), National Key Research and Development Program "major chronic non-infectious disease research" (2016YFC1303202), National Key Research and Development Program "precision medicine research" (2017YFC0908304).

\section{Footnote}

Reporting Checklist: The authors have completed the CARE reporting checklist. Available at http://dx.doi.org/10.21037/ tcr-20-1400
Conflicts of Interest: All authors have completed the ICMJE uniform disclosure form (available at http://dx.doi. org/10.21037/tcr-20-1400). The authors have no conflicts of interest to declare.

Ethical Statement: The authors are accountable for all aspects of the work in ensuring that questions related to the accuracy or integrity of any part of the work are appropriately investigated and resolved. All procedures performed in studies involving human participants were in accordance with the ethical standards of the institutional and/or national research committee(s) and with the Helsinki Declaration (as revised in 2013). Written informed consent was obtained from the patient.

Open Access Statement: This is an Open Access article distributed in accordance with the Creative Commons Attribution-NonCommercial-NoDerivs 4.0 International License (CC BY-NC-ND 4.0), which permits the noncommercial replication and distribution of the article with the strict proviso that no changes or edits are made and the original work is properly cited (including links to both the formal publication through the relevant DOI and the license). See: https://creativecommons.org/licenses/by-nc-nd/4.0/.

\section{References}

1. Guggenheim DE, Shah MA. Gastric cancer epidemiology and risk factors. J Surg Oncol 2013;107:230-6.

2. Sasako M, Sano T, Yamamoto S, et al. D2 lymphadenectomy alone or with para-aortic nodal dissection for gastric cancer. $\mathrm{N}$ Engl J Med 2008;359:453-62.

3. Kodera Y. Neoadjuvant chemotherapy for gastric adenocarcinoma in Japan. Surg Today 2017;47:899-907.

4. NCCN Clinical Practice Guidelines in Oncology. Gastric Cancer. (Version 2.2018).

5. Yamada Y, Higuchi K, Nishikawa K, et al. Phase III study comparing oxaliplatin plus S-1 with cisplatin plus S-1 in chemotherapy-naïve patients with advanced gastric cancer. Ann Oncol 2015;26:141-8.

6. Bando H, Yamada Y, Tanabe S, et al. Efficacy and safety of S-1 and oxaliplatin combination therapy in elderly patients with advanced gastric cancer. Gastric Cancer 2016;19:919-26.

7. Ito S, Sano T, Mizusawa J, et al. A phase II study of preoperative chemotherapy with docetaxel, cisplatin, and S-1 followed by gastrectomy with D2 plus para-aortic 
lymph node dissection for gastric cancer with extensive lymph node metastasis: JCOG1002. Gastric Cancer 2017;20:322-31.

8. Li J, Qin S, Xu J, et al. Randomized, double-blind, placebo-controlled Phase III trial of Apatinib in patients with chemotherapy-refractory advanced or metastatic adenocarcinoma of the stomach or gastroesophageal junction. J Clin Oncol 2016;34:1448-54.

9. Kanda T, Yajima K, Kosugi S, et al. Gastrectomy as a secondary surgery for stage IV gastric cancer patients who underwent S-1-based chemotherapy: a multi-institute retrospective study. Gastric Cancer 2012;15:235-44.

10. Katayama H, Tsuburaya A, Mizusawa J, et al. An integrated analysis of two phase II trials (JCOG0001 and JCOG0405) of preoperative chemotherapy followed by D3 gastrectomy for gastric cancer with extensive lymph node metastasis. Gastric Cancer 2019;22:1301-7.

11. Ishigami S, Natsugoe S, Nakajo A, et al. Salvage gastrectomy following a combination of biweekly paclitaxel and S-1 for stage IV gastric cancer. J Gastrointest Surg 2008;12:1370-5.

12. Sato $\mathrm{Y}$, Ohnuma H, Nobuoka T, et al. Conversion therapy for inoperable advanced gastric cancer patients by docetaxel, cisplatin, and S-1 (DCS) chemotherapy: a multi-institutional retrospective study. Gastric Cancer 2017;20:517-26.

13. Yoshikawa T, Omura K, Kobayashi O, et al. A phase II study of preoperative chemotherapy with S-1 plus cisplatin followed by D2/D3 gastrectomy for clinically serosapositive gastric cancer (JACCRO GC-01 study). Eur J Surg Oncol 2010;36:546-51.

14. Kosaka T, Akiyama H, Makino H, et al. Preoperative S-1 and docetaxel combination chemotherapy in patients with locally advanced gastric cancer. Cancer Chemother Pharmacol 2014;73:281-5.

15. KoizumiW, Takiuchi H, YamadaY, et al. Phase II study of oxaliplatin plus $\mathrm{S}-1$ as first-line treatment for advanced gastric cancer (G-SOX study). Ann Oncol 2010;21:1001-5.

16. Kim GM, Jeung HC, Sun YR, et al. A randomized phase II trial of S-1-oxaliplatin versus capecitabineeoxaliplatin in advanced gastric cancer. Eur J Cancer 2012;48:518-26.

Cite this article as: Zhang N, Deng J, Sun Y, Xiao J, Li H, Liang H. An unresectable gastric cancer with bulky lymph node metastases treated with SOX chemotherapy plus apatinib followed by D3 radical gastrectomy: a case report. Transl Cancer Res 2021;10(1):537-545. doi: 10.21037/tcr-20-1400
17. Satake H, Miki A, Kondo M, et al. Phase I study of neoadjuvant chemotherapy with $\mathrm{S}-1$ and oxaliplatin for locally advanced gastric cancer (Neo G-SOX PI). ESMO Open 2017;2:e00130.

18. Roviello G, Ravelli A, Polom K, et al. Apatinib: a novel receptor tyrosine kinase inhibitor for the treatment of gastric cancer. Cancer Lett 2016;372:187-91.

19. Zheng Y, Yang X, Yan C, et al. Effect of apatinib plus neoadjuvant chemotherapy followed by resection on pathologic response in patients with locally advanced gastric adenocarcinoma: a single-arm, open-label, phase II trial. Eur J Cancer 2020;130:12-9.

20. Kunisaki C, Takahashi M, Fukushima T, et al. The influence of stage migration on the comparison of surgical outcomes between D2 gastrectomy and D3 gastrectomy (para-aortic lymph node dissection): a multi-institutional retrospective study. Am J Surg 2008;196:358-63.

21. Sasako M. Role of surgery in multidisciplinary treatment for solid cancers. Int J Clin Oncol 2004;9:346-51.

22. Yoshikawa T, Sasako M, Sano T, et al. Stage migration caused by D2 dissection with para-aortic lymphadenectomy for gastric cancer form the results of a prospective randomized controlled trial. Br J Surg 2006;93:1526-9.

23. Mandard AM, Dalibard F, Mandard JC, et al. Pathologic assessment of tumor regression after preoperative chemoradiotherapy of esophageal carcinoma. Clinicopathologic correlations. Cancer 1994;73:2680-6.

24. Ruan H, Dong J, Zhou X, et al. Multicenter phase II study of apatinib treatment for metastatic gastric cancer after failure of second-line chemotherapy. Oncotarget 2017;8:104552-9.

25. Cheng H, Sun A, Guo Q, et al. Efficacy and safety of apatinib combined with chemotherapy for the treatment of advanced gastric cancer in the Chinese population: a systematic review and meta-analysis. Drug Des Devel Ther 2018;12:2173-83.

26. Dong S, Yu JR, Zhang Q, et al. Neoadjuvant chemotherapy in controlling lymph node metastasis for locally advanced gastric cancer in a Chinese population. J Chemother 2016;28:59-64. 